\title{
IBRAHITHM: New effective and flexible Algorithm for the division of numbers and polynomials
}

\author{
T Ibrahim $^{1}$ \\ ${ }^{1}$ Mansoura University
}

May 8, 2020

\begin{abstract}
For a long time we study the long division of numbers and polynomials and perform it from left to right as we know . In this paper we introduce new flexible and effective manual method for the division of numbers and polynomials which perform from right to left which we call it IBRAHITHM Division". The method is easy and it has done without any problems when we divide numbers on an odd number except five and its multiplies. But we have some obstacles when we divide numbers by even numbers or a number five and its multiplies in the decimal system. The method can be use for all number systems such as decimal, binary, octal and hexadecimal Systems. Also we use this method to divide numbers with decimal fractions . We can use it to find the square roots of numbers. Finally we have built IBRAHITHM polynomial division and IBRAHITHM synthetic division. Our method here is characterized by the fact that it performs division and test the divisibility at the same time.Finally we introduce the relation between the standard algorithm division method and IBRAHITHM division method .
\end{abstract}

\section{Hosted file}

Paper.pdf available at https://authorea.com/users/315205/articles/449415-ibrahithm-neweffective-and-flexible-algorithm-for-the-division-of-numbers-and-polynomials 